\title{
Relation between Mycoplasmas and Leukaemia and Related Diseases
}

\author{
R. J. FALLON, ${ }^{*}$ M.D., B.SC., M.C.PATH. ; DOROTHY K. JACKSON, $\dagger$ B.SC.
}

\begin{abstract}
Cummary: Forty-five bone marrow specimens from leukaemia patients and $\mathbf{4 0}$ marrows from nonleukaemia patients were cultured for mycoplasmas. No mycoplasmas were isolated. Sera from patients with leukaemia or reticuloses and from non-leukaemic subjects were examined for antibodies to the Negroni and $\mathbf{8 8 0}$ strains of Mycoplasma pulmonis and also to the K7, K10, and prototype (PG18) strains of $M$. fermentans. No significant differences were observed between the two groups of patients with respect to antibodies to these mycoplasmas. These findings support those of the majority of other workers in failing to show any relationship between mycoplasmas and neoplastic disease in man.
\end{abstract}

\section{Introduction}

Negroni (1964) reported the isolation of agents from tissue cultures inoculated with bone marrow from leukaemic patients. The agents appeared to be identical with each other, and were later shown (Fallon et al., 1965) to be a strain of Mycoplasma pulmonis - a rodent pathogen. Negroni (1964) and Beale et al. (1968) also showed that sera from patients with leukaemia neutralized the agents in tissue culture. This finding was not confirmed by Grist (1966).

Grace et al. (1965) reported the isolation of four antigenically identical cytopathic agents from tissue cultures inoculated with leukaemic or lymphomatous tissue. These were recognized as mycoplasmas in 1961, and the representative strain, designated 880 , was shown (Leach and Butler, 1966) to be a strain of M. pulmonis.

Murphy et al. (1965) reported the isolation of mycoplasmas from tissue cultures inoculated with bone marrow from leukaemic patients and also, using a special culture medium, the direct isolation of mycoplasmas from leukaemic material. The mycoplasmas which they isolated were related to $M$. fermentans, an organism originally isolated from the human genital tract.

Hayflick and Koprowski (1965) and Barile et al. (1966) have reported the isolation of $M$. orale type 1 , a human oral strain of mycoplasma, from bone marrow inoculated directly into mycoplasma medium.

In view of Negroni's report it was decided to attempt the direct isolation of mycoplasmas from leukaemic bone marrow and also to search for antibodies not only to the Negroni strain of $M$. pulmonis but also to the 880 strain in case this differed serologically from the Negroni strain. Furthermore, antibodies were sought to the $\mathrm{K} 7$ and $\mathrm{K} 10$ strains of $M$. fermentans isolated by Murphy et al. (1965) as well as to the prototype strain of $M$. fermentans. In order to assess the significance of the findings in leukaemic patients sera from non-leukaemic subjects were also examined. Two serological tests (complement fixation and metabolic inhibition) were used in order to increase the chance of detecting antibodies to the various strains of mycoplasmas. * Consultant in Labiratory Medicine, Ruchill Hospital, Glasgow N.W. ;
Honorary Lecturer in Bacteriology, University of Glasgow.

† Research Assistant, Pathology Department, Ruchill Hospital, Glasgow N.W.

\section{Materials and Methods}

\section{Bone Marrow Specimens}

The bone marrow specimens were usually placed directly into fluid Hayflick's medium (Chanock et al., 1962), but a small number were heparinized and then inoculated not only into fluid Hayflick's medium but also on to solid medium and on to a medium containing human serum and vitamin supplements (Murphy et al., 1965). Usually $5 \mathrm{ml}$. of broth (in later specimens with a slope of solid medium in the bottle) was inoculated, but varying volumes were used in order to try to avoid suppression of growth by any inhibitory factors that might have been present in the marrow. All specimens were inoculated into broth for incubation under both aerobic and anaerobic conditions, but because many of the cultures were inoculated at another hospital some specimens were incubated aerobically at $37^{\circ} \mathrm{C}$. for up to 48 hours before anaerobic cultures were set up. Cultures in turn were passaged serially at weekly intervals until each specimen had passed through six blind passages both aerobically and anaerobically. Forty-eight hours after the initial culture had been set up and at each passage fluid medium was also inoculated on to solid medium for aerobic and anaerobic incubation. Cultures on solid medium were kept at least one week after inoculation, usually longer. The last 13 specimens examined were subcultured in fluid and on solid Murphy's medium as well as in Hayflick's medium.

The media used were shown to be capable of supporting the growth of stock strains of mycoplasmas as well as of freshly isolated strains. Plates were examined under 45 and 150 diameters magnification in order that even colonies of the size produced by $\mathrm{T}$-strain mycoplasmas should not be missed.

The 45 leukaemic marrows examined came from both acute untreated and chronic cases as well as from treated cases and those in remission. Twenty-seven came from children under the age of 14 . The 40 marrows from non-leukaemic patients came almost exclusively from adults.

\section{Mycoplasma Strains}

The Negroni strain of $M$. pulmonis was that isolated in this laboratory (Grist and Fallon, 1964). The 880 strain was obtained from Dr. J. T. Grace, of Buffalo, $M$. fermentans strains $\mathrm{K} 7$ and $\mathrm{K} 10$ were obtained from Dr. W. H. Murphy, of Ann Arbor, and strain PG18 from Dr. Sheila Stewart, of Edinburgh.

\section{Complement Fixation Tests}

Complement fixation tests were performed in plastic trays, the technique of Bradstreet and Taylor (1962) as modified by Grist et al. (1966) being used.

Antigens and rabbit antisera were prepared as described by Fallon and Jackson (1967). Latterly a Spinco model L ultracentrifuge was used for preparing antigens, the mycoplasmas being deposited at $35,000 \mathrm{~g}$ for 45 minutes in the 21 rotor and then being washed in phosphate-buffered saline and deposited by centrifuging at $26,500 \mathrm{~g}$ for 35 minutes in the 
40 rotor. Mycoplasmas were washed thrice before sonication. Difficulty was experienced with $M$. fermentans strains in that antigens tended to be anticomplementary. Attempts to render the antigens less anticomplementary by heating, absorbing with complement, or extraction by the method of Kenny and Grayston (1965) were unsuccessful. Sera showing any fixation in a screening test were titrated in a chessboard fashion against antigen. Only complete fixation was recorded in the latter tests.

\section{Metabolic Inhibition Tests}

Metabolic inhibition tests were performed in microtitre trays by the method of Taylor-Robinson et al. (1966a).

\section{Sera}

Cases of Leukaemia or Reticulosis.-The sera examined were obtained from 125 cases of leukaemia or reticulosis (for details see Table I). Sera were obtained from patients in all stages of disease, including treated and untreated cases.

Non-leukaemic Subjects. - Sera were obtained from two main sources: (1) 287 patients admitted to Ruchill Hospital with conditions other than leukaemia, and (2) 112 blood donors.

For complement fixation tests sera were tested at a dilution of $1 / 8$, whereas for metabolic inhibition tests the dilutions were of $1 / 4,1 / 8$, and $1 / 16$. Three dilutions of serum were used in metabolic inhibition tests in case the high concentration of serum in the cup containing the $1 / 4$ dilution of serum potentiated the growth of mycoplasmas, thus obscuring the inhibitory effect of any antibody which may have been present. Sera showing a titre of $1 / 16$ in these tests were titrated out. For both tests sera were inactivated by heating at $56^{\circ}$ C. for 30 minutes.

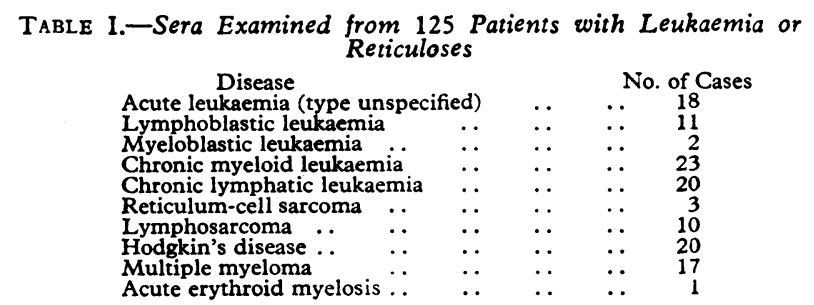

As only very small amounts of some sera were available not all were tested against all the antigens. In addition some sera were anticomplementary. These two facts explain why the denominators in the various tables were not uniform.

In view of the inhibitory effect of some antibiotics on mycoplasmas a careful check was made on whether any serum giving a positive result in a metabolic inhibition test was obtained at a time when the patient was receiving an antibiotic, such as chloramphenicol, tetracycline, or an aminogylcoside, known to be active against mycoplasmas. Any such doubtful positive result was excluded from the analysis of results.

\section{Results}

Bone Marrow Culture.-No mycoplasmas were isolated from any of the bone marrow examined.

\section{Serological Studies}

Antibodies were sought by means of two distinct groups of antigen: (1) M. pulmonis strains-the Negroni and strain 880 mycoplasmas, and (2) $M$. fermentans strains-the prototype strain (PG18) and strains $\mathrm{K} 7$ and $\mathrm{K} 10$. The results of com- plement fixation tests with strains of $M$. pulmonis are shown in Table II. It will be seen that no serum gave a positive result.

\begin{tabular}{l} 
TABLE II.-Complement Fixation \\
\hline \multirow{2}{*}{ Sera } \\
\end{tabular}

Numerator $=$ Number of sera positive at a dilution of $1 / 8$ or zreater. Denominator $=$ Total tested.

The results of complement fixation tests with the varioue strains of $M$. fermentans are shown in Table III. Of the leukaemic sera giving a positive result, with $M$. fermentans group antigens two reacted with PG18, K7, and $\mathrm{K} 10$ antigens. Similarly the two non-leukaemic sera which fixed complement with PG18 also fixed complement with $\mathrm{K} 7$ and $\mathrm{K} 10$ antigens.

TABLE III.-Complement Fixation Tests with Strains of M. fermentans

\begin{tabular}{cc|c|c|c}
\hline \multirow{2}{*}{ Sera } & \multicolumn{3}{|c}{ M. fermentans Strains } \\
\cline { 2 - 5 } & & PG18 & K7 & K10 \\
\hline $\begin{array}{llll}\text { Leukaemia and reticulosis } \\
\text { Non-leukaemic }\end{array}$ & $\ldots$ & $3 / 88(3 \cdot 4)$ & $2 / 87(2 \cdot 3)$ & $2 / 87(2 \cdot 3)$ \\
&.. & $2 / 151(1 \cdot 3)$ & $4 / 151(2 \cdot 6)$ & $3 / 147(2 \cdot 0)$ \\
\hline
\end{tabular}

Numerator $=$ Number of sera positive at a dilution of $1 / 8 . \quad$ Denominator $=$ Total sera tested. Percentages are given in parentheses.

The cross-reaction of sera with $M$. fermentans antigens $\mathrm{PG} 18, \mathrm{~K} 7$, and $\mathrm{K} 10$ is to be expected, as the mycoplasma complement fixation test is species-specific. Hence when the cross-reactions of rabbit antisera to these mycoplasmas prepared by the method previously described (Fallon and Jackson, 1967) are considered (Table IV) it can be seen that there was not a great difference in titre whether homologous or heterologous $M$. fermentans antigens were used in the test, except in the case of antiserum to $\mathrm{K} 10$ reacting with antigen prepared from the PG18 strain of $M$. fermentans.

TABLE IV.-Complement Fixation Tests with Strains of M. fermentans

\begin{tabular}{c|c|c|c}
\hline \multirow{3}{*}{ Antisera } & \multicolumn{3}{|c}{ Antigen } \\
\cline { 2 - 4 } & PG18 & K7 & K10 \\
\hline PG18 & 256 & 128 & 123 \\
K7 & 128 & 258 & 255 \\
K10 & 64 & 1,021 & 1,024
\end{tabular}

Figures are the reciprocal of the serum dilution.

The results of metabolic inhibition tests for antibodies to $M$. pulmonis are shown in Table V. Only one subject was shown to possess antibodies, and these were to the Negroni mycoplasma but not to strain 880 . This reaction with only one of the two $M$. pulmonis antigens would accord with our previous observation (Fallon and Jackson, 1967) that the metabolic inhibition test tends to be strain-specific.

TABLE V.-Metabolic Inhibiton Tests with Strains of $M$. pulmonis

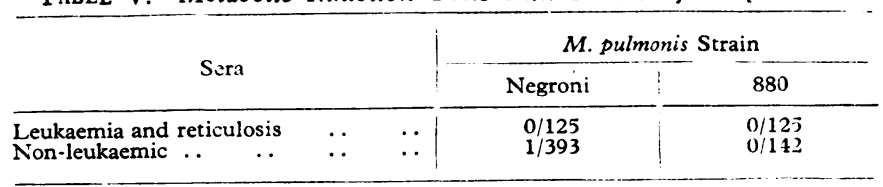

Numerator $=$ Number of sera positive at a dilution of $1 / 4$ or greater. DenominaNumerator $=$ Number
tor $=$ Number tested.

The results of metabolic inhibition tests for antibodies to $M$. fermentans strains are shown in Table VI. Only one of the sera reacted with more than one antigen, this being serum from a blood donor which reacted with both $\mathrm{K} 7$ and $\mathrm{K} 10$ antigens. Serum titres usually ranged from $1 / 4$ to $1 / 16$, but two sera, one from a patient with Hodgkin's disease and the other from a blood donor, had titres of $1 / 64$. 
TABLE VI.-Metabolic Inhibition Tests with Strains of M. fermentans

\begin{tabular}{|c|c|c|c|c|}
\hline \multirow{2}{*}{\multicolumn{2}{|c|}{ Sera }} & \multicolumn{3}{|c|}{ M. fermentans Strain } \\
\hline & & PG18 & K7 & K10 \\
\hline $\begin{array}{l}\text { Leukaemia and reticulosis } \\
\text { Non-leukaemic }\end{array}$ & $\therefore$ & $\begin{array}{l}1 / 125(0.8) \\
4 / 198(2 \cdot 0)\end{array}$ & $\begin{array}{l}3 / 125(2 \cdot 4) \\
4 / 199(2 \cdot 0)\end{array}$ & $\begin{array}{l}2 / 125(1.6) \\
1 / 199(0.5)\end{array}$ \\
\hline
\end{tabular}

Numerator $=$ Number of sera positive at a dilution of $1 / 4$ or greater. Denominator $=$ Total number tested. Percentages are given in parentheses.

\section{Discussion}

No bone marrow specimen yielded mycoplasmas. This finding does not preclude the possibility that these marrow specimens contained mycoplasmas which could not be cultivated by the technique described. However, our experience is similar to that of Sutton (1966), Butler et al. (1967), Lazarus et al. (1967), and Tobin and Jones (1967), but contrasts sharply with that of Murphy et al. (1965, 1967), and Barile et al. (1966), who succeeded in isolating mycoplasmas directly from the marrow of a number of patients. The significance of the isolation of mycoplasmas from bone marrow has been interpreted with great caution by those workers who have reported positive isolations, and does not necessarily bear any causal relation to the disease in the patients from whom the mycoplasmas were isolated, but may represent a break-down in normal defence mechanisms in these patients.

There was no demonstrable relation between antibodies to mycoplasmas and disease, nor was there any evidence that the group of patients with leukaemia or reticulosis had a lower prevalence of antibodies than the non-leukaemic subjects.

All the positive results reported were produced on several occasions in complement fixation tests by "chessboard" titration and in metabolic inhibition tests by repeating the tests. These latter repeated tests eliminated a number of sera with initial possible low-level (1/4) reactions. It is possible that these sera contained low levels of antibody that were lowered still further by freezing and thawing; but this remains uncertain, and such lowering of titre has not been seen in this laboratory with hyperimmune rabbit sera frozen and thawed 10 times (Forshaw, unpublished). Furthermore, the occurrence of low-level non-reproducible reactions was the same in sera stored for a short time as in 91 of the reticulosis sera which were originally studied several years previously by Heath et al. (1964). However, the elimination of doubtiul positives in this way by repeated tests may be one reason why the proportion of patients with antibodies to $M$. fermentans was lower than that reported by Taylor-Robinson et al. (1966a). On the other hand, it may represent a real difference between the populations studied.

Care was taken to ensure that false-positive results in the metabolic inhibition tests due to the presence of tetracycline or other antibiotics in patients' sera were excluded from the final analysis. This factor was not taken into account by Negroni (1964) in the analysis of the results of neutralization tests with sera from patients with leukaemia because at that time the "agent" was thought to be a virus. No mention of the presence or absence of antibiotics in patients' sera was made in the more recent report by Beale et al. (1968), though these workers were careful to point out that the inhibitor in the sera they examined had not been shown to be antibody.

None of the 23 children (16 leukaemic, 7 non-leukaemic) whose sera were tested against all the antigens showed the presence of antibody. This is not surprising, as antibodies to another mycoplasma, $M$. pneumoniae, have been shown to be less prevalent in children than in adults (Suhs and Feldman, 1966 ; Taylor-Robinson et al., 1966b).

Several studies have now been published where the sera of normal and leukaemic subjects have been examined for mycoplasma antibodies. Murphy et al. (1967) used fresh unheated sera in neutralization tests against the K7 and K10 strains of tissue culture. These tests differed from those reported in this paper in that we used sera inactivated at $56^{\circ} \mathrm{C}$. for 30 minutes ; furthermore, no mention was made by Murphy et al. of the exclusion of false-positive results due to antibiotics. The results of Murphy et al. cannot therefore be compared with those reported here.

Millian and Spigland (1966), using the indirect haemagglutination test, examined sera from normal subjects and from patients with Hodgkin's disease, lymphosarcoma, reticulumcell sarcoma, and chronic lymphatic leukaemia. They looked for antibodies to human-associated mycoplasmas, including $M$. fermentans, as well as to the 880 strain of $M$. pulmonis and another rodent pathogen, $M$. arthritidis (" $M$. hominis type 2 "). No significant differences were found between the leukaemic and non-leukaemic subjects with respect to antibodies to any of the mycoplasmas used as antigens. It is interesting to note that the proportion of subjects with antibodies to $M$. fermentans found by Millian and Spigland $(72 \%$ of leukaemic subjects, $82 \%$ of controls) was much higher than that found in the present study or in that of Taylor-Robinson et al. (1966a). This may be due to the high sensitivity of the indirect haemagglutination technique, which itself could account for the demonstration of Millian and Spigland of antibodies to the 880 strain of $M$. pulmonis in a small proportion of individuals. In any case the origin of antibodies to $M$. fermentans remains an enigma in view of the complete lack of evidence of its pathogenicity together with the low frequency with which it is isolated.

Our results and those of Grist (1966) do not agree with the findings of Negroni (1964) and of Beale et al. (1968), and we have been unable to demonstrate the presence of antibody to the Negroni strain of $M$. pulmonis in the sera of patients with leukaemia. Some possible reasons for this have been noted above; however, negative results must be interpreted with caution. There is no reason why contact with mycoplasma should result in the production of antibody, and indeed it has been shown by Jensen et al. (1967) that the pathogen $M$. pneumoniae could be isolated from patients with clinical disease who failed to produce antibody detectable by three different tests. Furthermore, mycoplasmas have been shown to produce changes in chromosomes in cells in tissue culture (Fogh and Fogh, 1965'; Paton et al., 1965), and transformation of cells has also been observed (Macpherson and Russell, 1966), so that these organisms would appear to have some unusual properties which could possibly be related to the onset of a neoplastic process. Nevertheless, our findings agree with those of the majority of other workers in failing to show any relation between mycoplasmas and neoplastic disease in man.

This work was made possible by a generous grant from the Secretary of State for Scotland on the recommendation of the Advisory Committee on Medical Research. Most of the leukaemic sera from adults were provided by Dr. R. B. Heath, of St. Bartholomew's Hospital. The leukaemic marrows and sera from children were supplied by Dr. M. L. N. Willoughby, of the Royal Hospital for Sick Children, Glasgew. Other specimens were provided by Professor A. S. Douglas and Drs. J. Craik, J. L. Markson, and J. Whitelaw. The assistance of Miss K. A. Forshaw, Mr. D. Hutchinson, and Mr. D. Marshall is gratefully acknowledged.

\section{REFERENCES}

Barile, M. F., Bodey, G. P., Snyder, J., Riggs, D. B., and Grabowski, M. W. (1966). F. nat. Cancer Inst., 36, 155 .

Beale, A. J., Christofinis, G. J., and Negroni, G. (1968). Brit. med. F., 1, 580 .

Bradstreet, C. M. P., and Taylor, C. E. D. (1962). Mth. Bull. Minist. Hlth Lab. Serv., 21, 96.

Butler, M., Fairley, G. H., and Leach, R. H. (1967). Brit. med. 7., 2, 243.

Chanock, R. M., Hayflick, L., and Barile, M. F. (1962). Proc. nat. Acad. Sci., 48, 41 .

Fallon, R. J., Grist, N. R., Inman, D. R., Lemcke, R. M., Negroni, G., and Woods, D. M. (1965). Brit. med. F., 2, 388.

Fallon, R. J., and Jackson, D. K. (1967). Lab. Anim., 155. 
Fogh, J. and Fogh, H. (1965). Proc, Soc exp. Biol. (N.Y.), 119, 233.

Grace, J. T., Horoszewicz, J. S., Stim, T. B., Mirand, E. A., and James, C. (1965). Cancer (Philad.), 18, 1369

Grist, N. R. (1966). Brit. med. '., 2, 1135.

Grist, N. R., and Fallon, R. J. (1964). Brit. med. 7., 2, 1263.

Grist, N. R., Ross, C. A. C., Bell, E. J., and Stott, E. J. (1966). Diagnostic Methods in Clinical Virology. Oxford.

Hayflick, L., and Koprowski, H. (1965). Nature (Lond.), 205, 713.

Heath, R. B., Fairley, G. H., and Malpas, J. S. (1964).' Brit. F. Haemat., 10, 365 .

Jensen, K. J., Senterfit, L. B., Scully, W. E., Conway, T. J., West, R. F., and Drummy, W. W. (1967). Amer. F. Epidem., 86, 419.

Kenny, G. E., and Grayston, J. T. (1965). F. Immunol., 95, 19.

Lazarus, H., Beckett, K. M. K., Cuppels, D., and Foley, G. E. (1967). 7. Bact., 94, 1797.

Leach, R. H., and Butler, M. (1966). 7. Bact., 91, 934
Macpherson, I., and Russell, W. (1966). Nature, (Lond.), 210, 1343.

Millian, S. J., and Spigland, I. (1966). Cancer (Philad.), 19, 1820.

Murphy, W. H., Ertel, I. J., Bullis, C., and Zarafonetis, C. J. D. (1967). Proc. Soc. exp. Biol. (N.Y.), 124, 366.

Murphy, W. H., Ertel, I. J., and Zarafonetis, C. J. D. (1965). Cancer (Philad.), 18, 1329.

Negroni, G. (1964). Brit. med. 7., 1, 927.

Paton, G. R., Jacobs, J. P., and Perkins, F. T. (1965). Nature (Lond.), 207, 43.

Suhs, R. H., and Feldman, H. A. (1966). Amer. F. Epidem., 83, 357.

Sutton, R. N. P. (1966). Brit. Med. F., 2, 1496 .

Sutton, R. N. P. (1966). Brit. Med. H. W, 1496. C and Chanock, R. M (1966a). F. Hyg. (Lond.), 64, 91.

Taylor-Robinson, D., Shirai, A., Soběslavský, O., and Chanock, R. M. (1966b). Amer. F. Epidem., 84, 301.

Tobin, B. M., and Jones, D. M. (1967). Brit. med. F., 1, 758.

\title{
Studies in the Epidemiology of Tinea Pedis. IX: Tinea Pedis and Erythrasma in New Patients at a Chiropody Clinic
}

\author{
MARY P. ENGLISH,* M.SC. ; J. TURVEY, $\dagger$ M.CH.S., s.R.CH.
}

\begin{abstract}
Summary : The feet of 259 new patients at a chiropody $\checkmark$ clinic were examined for tinea pedis, onychomycosis, and erythrasma : $23 \%$ of men and $4 \%$ of women were infected by dermatophytes, and the nails of seven males were infected by non-dermatophytes. Of 200 patients examined under Wood's light $37 \%$ showed the coral-red fluorescence of erythrasma.

Of the 259 patients, $9.7 \%$ were infected by Trichophyton interdigitale, $2.7 \%$ by $T$. rubrum, and $1.5 \%$ by Epidermophyton floccosum. Reasons are given, based on the method of selection of the patients, for supposing that $T$. interdigitale is still the dominant cause of tinea pedis in the population at large, despite figures from dermatological clinics suggesting the dominance of $T$. rubrum. The high incidence of infection in males compared with females corresponds with similar findings in schoolchildren.
\end{abstract}

\section{Introduction}

The great majority of cases of tinea pedis are mild, and are treated by the patient himself or by the general practitioner. Yet almost all published figures for its incidence, and for the incidence of the fungi causing it, have been based on persons attending skin clinics who have the disease sufficiently severely to have been referred to these specialist centres. All figures so obtained are bound to be biased towards a high total incidence and towards the species of fungus causing the most severe, widespread, and recalcitrant lesions. The nearest approach to studies of the ordinary population have been those carried out among children in day schools; for instance, in this country, by English and Gibson (1959). Nothing is known of the incidence of the disease, or of the fungi causing it, in the adult population at large, mainly because of the difficulty of accurate sampling and the magnitude of the task.

It had been observed by one of us (J. T.) that very few people attending a chiropodist come with an initial complaint of tinea pedis. Such people, therefore, while admittedly not constituting a random sample of the adult population, approach such a sample closely enough to provide a practical substitute for an otherwise impracticable undertaking. It was chiefly for this reason that the present survey of new patients attending a chiropody clinic was begun.

* Mycologist, General Hospital, Bristol 1

+ Senior Lecturer, Chelsea School of Chiropody, London N.W.8.

\section{Subjects}

The subjects comprised new patients seen by one of us (J.T.) at a weekly clinic held only during term-time at the Chelsea School of Chiropody. Many more women than men attended the clinic; therefore, to obtain the required numbers of each sex, both men and women were included from 8 October 1964 to 29 April 1965, and men only were examined from 8 October 1965 to 10 February 1966 and from 27 October 1966 to 18 May 1967. Though men were examined over a much longer period than women this is unlikely to have distorted our results, as they were always seen at the same times of year.

After our survey was complete Koch (1967) pointed out that in Erfurt (Germany) chiropody clinics themselves were the most prolific source of cross-infection with tinea pedis of the various sports and cosmetic establishments examined by him. No such investigation has been carried out in British chiropody clinics, but Koch's findings do not invalidate our results, as all our subjects were new patients.

Scrapings were taken by J. T. from all scaling, erythematous, macerated, pustular, or cracked iesions of the foot, and clippings were obtained from any dystrophied or discoloured toe-nails, unless clearly of non-mycotic origin. In lesion-free patients scrapings were taken from between the fourth and fifth toes. As all patients with lesions elsewhere than in the toe-wébs also had them on the latter site, scrapings were, in fact, obtained from the toe-webs of every patient. It has been pointed out by numerous workers-for example, Gentles and Holmes (1957)-that the incidence of tinea-like lesions on the feet far exceeds the incidence of true fungal infection. This was also true in this survey, but the fact is so well known that figures for the total incidence of lesions are omitted.

All clinical material was posted to Bristol for laboratory examination, which was carried out by M.P.E.

All the women and the first 102 men were screened for interdigital erythrasma by examination of their toe-webs for coralred fluorescence under Wood's light.

Relevant particulars of each patient were filled in on a questionary prepared in such a way that the replies could be used for punched card analysis without further coding.

\section{Laboratory Methods}

The laboratory methods used for fungi were the standard methods of microscopy and culture used previously in this 